Claremont Colleges

Scholarship@ Claremont

All HMC Faculty Publications and Research

HMC Faculty Scholarship

6-12-2003

\title{
Solitary Waves in Layered Nonlinear Media
}

\author{
Randall J. Leveque \\ University of Washington - Seattle Campus \\ Darryl H. Yong \\ Harvey Mudd College
}

\section{Recommended Citation}

Leveque, RJ, Yong, DH. Solitary waves in layered nonlinear media. J App Math. 2003;63(5): 1539-1560.

This Article is brought to you for free and open access by the HMC Faculty Scholarship at Scholarship @ Claremont. It has been accepted for inclusion in All HMC Faculty Publications and Research by an authorized administrator of Scholarship @ Claremont. For more information, please contact scholarship@cuc.claremont.edu. 


\title{
SOLITARY WAVES IN LAYERED NONLINEAR MEDIA*
}

\author{
RANDALL J. LEVEQUE ${ }^{\dagger}$ AND DARRYL H. YONG
}

\begin{abstract}
We study longitudinal elastic strain waves in a one-dimensional periodically layered medium, alternating between two materials with different densities and stress-strain relations. If the impedances are different, dispersive effects are seen due to reflection at the interfaces. When the stress-strain relations are nonlinear, the combination of dispersion and nonlinearity leads to the appearance of solitary waves that interact like solitons. We study the scaling properties of these solitary waves and derive a homogenized system of equations that includes dispersive terms. We show that pseudospectral solutions to these equations agree well with direct solutions of the hyperbolic conservation laws in the layered medium using a high-resolution finite volume method. For particular parameters we also show how the layered medium can be related to the Toda lattice, which has discrete soliton solutions.
\end{abstract}

Key words. nonlinear elasticity, solitons, layered media, homogenization, Toda lattice

AMS subject classifications. 74J35, 74Q10, 35B27, 35L65, 37K60

DOI. $10.1137 /$ S0036139902408151

1. Introduction. Consider a heterogeneous medium composed of alternating layers of two different materials labeled $\mathrm{A}$ and $\mathrm{B}$. The layers have widths $\delta_{A}=\alpha \delta$ and $\delta_{B}=(1-\alpha) \delta$, repeating periodically with period $\delta$. The densities of the two materials are $\rho_{A}$ and $\rho_{B}$, respectively, and their response to compression or expansion is characterized by the stress-strain relations $\sigma_{A}(\epsilon)$ and $\sigma_{B}(\epsilon)$. Then compressional waves propagating in the direction of layering are modeled by the one-dimensional hyperbolic system of conservation laws

$$
\begin{aligned}
\epsilon_{t}(x, t)-u_{x}(x, t) & =0 \\
(\rho(x) u(x, t))_{t}-\sigma(\epsilon(x, t), x)_{x} & =0
\end{aligned}
$$

where $\epsilon(x, t)$ is the strain and $u(x, t)$ the velocity. For the layered medium we have

$$
(\rho(x), \sigma(\epsilon, x))= \begin{cases}\left(\rho_{A}, \sigma_{A}(\epsilon)\right) & \text { if } j \delta<x<(j+\alpha) \delta \text { for some integer } j \\ \left(\rho_{B}, \sigma_{B}(\epsilon)\right) & \text { otherwise }\end{cases}
$$

For sufficiently small strains, the response can be modeled by linear constitutive relations

$$
\sigma_{A}(\epsilon)=K_{A} \epsilon, \quad \sigma_{B}(\epsilon)=K_{B} \epsilon,
$$

where the bulk moduli $K_{A}$ and $K_{B}$ of both materials are constants. Waves having long wavelength relative to the layer width can be modeled by a homogenized linear PDE that has the form of a wave equation with small dispersive term. The effective wave speed and dispersion coefficient can be calculated from the above parameters describing the layered medium. This linear case is reviewed in section 2.

\footnotetext{
*Received by the editors May 22, 2002; accepted for publication (in revised form) October 16, 2002; published electronically June 12, 2003. This work was supported in part by DOE grant DEFG03-00ER25292 and NSF grants DMS-9803442 and DMS-0106511.

http://www.siam.org/journals/siap/63-5/40815.html

$\dagger$ Department of Applied Mathematics, University of Washington, Box 352420, Seattle, WA 981952420 (rjl@amath.washington.edu).

$\ddagger$ Department of Applied and Computational Mathematics, California Institute of Technology, 1200 E. California Blvd., MC 217-50, Pasadena, CA 91125 (dyong@caltech.edu).
} 
More interesting behavior is observed when the constitutive relations are nonlinear in each layer. In this case a long-wavelength pulse breaks up into a series of solitary waves that are each only a few layers wide. This is not a complete surprise since we expect that a nonlinear wave equation with a dispersive term (again arising from the layering) may give rise to soliton-like solutions, and the classic soliton equations such as the KdV equation also exhibit this type of behavior. However, the waves appearing in the layered medium are harder to characterize than classical solitary waves. The wave shape is constantly modulating as it passes through the layers, and thus it cannot be expressed in the form of a fixed-shape wave propagating at constant speed. These waves appear to interact as solitons, essentially passing through one another with at most a shift in phase, but it is not yet clear to what extent they are truly solitons in the technical sense.

To the best of our knowledge, these waves were first observed computationally in [4], where a high-resolution finite volume method is presented that calculates accurate approximations to these waves. This method is also described in [1]. Here we use this method to further explore the nature of these waves.

Solitary waves in nonlinear elastic bars have been observed and studied in the past; see, for example, [5] and the references therein. In this case the material is homogeneous but the finite cross-sectional area gives rise to reflections at the tractionfree boundaries and hence dispersion.

In section 4 we show that a particular choice of the layered medium can be directly related to the Toda lattice. This may be significant since the Toda lattice is completely integrable and has discrete soliton solutions that can be compared directly to the solitary waves we observe in the corresponding layered medium.

In section 5 we present a homogenized set of equations for the nonlinear layered medium, and show that solutions to this system agree well with solutions to the layered medium equations. These equations contain dispersive terms and more complicated nonlinearities.

2. Waves in linear media. For a homogeneous linear medium with constant material parameters $\rho$ and $K$, the governing equations (1.1) are simply

$$
\begin{aligned}
\epsilon_{t}-u_{x} & =0, \\
\rho u_{t}-K \epsilon_{x} & =0
\end{aligned}
$$

or $q_{t}+A q_{x}=0$, where

$$
q=\left[\begin{array}{c}
\epsilon \\
\rho u
\end{array}\right], \quad A=-\left[\begin{array}{cc}
0 & 1 / \rho \\
K & 0
\end{array}\right] .
$$

The eigenvalues of $A$ are $\lambda^{1}=-c$ and $\lambda^{2}=+c$, where $c=\sqrt{K / \rho}$ is the speed of sound in the material. Purely leftgoing or rightgoing waves have $q(x, t)$ proportional to the corresponding eigenvector $r^{1}$ or $r^{2}$, respectively, given by

$$
r^{1}=\left[\begin{array}{l}
1 \\
Z
\end{array}\right] \quad \text { and } \quad r^{2}=\left[\begin{array}{c}
1 \\
-Z
\end{array}\right] \text {. }
$$

Here $Z=\rho c=\sqrt{K \rho}$ is the impedance of the material.

In a layered linear medium, wave propagation can be more complicated. If the layers are impedance-matched, $Z_{A}=Z_{B}$, then a rightgoing wave (for example) has the same characteristic form in both layers, and the wave simply has a different speed 
in each layer. It will distort as it speeds up and slows down, but remains entirely rightgoing and moves with an effective velocity

$$
\bar{c}=\left(\frac{\alpha}{c_{A}}+\frac{(1-\alpha)}{c_{B}}\right)^{-1}
$$

as is easily verified by computing the time required to cross two adjacent layers.

If $Z_{A} \neq Z_{B}$, on the other hand, then waves are partially reflected at each interface. It is impossible to have a purely rightgoing wave in such a composite material. However, a wave with long wavelength relative to the scale of the layers (i.e., wavelength $\gg \delta)$ can appear to be essentially rightgoing, and propagates at an effective velocity of

$$
\bar{c}=\sqrt{\hat{K} / \bar{\rho}},
$$

where

$$
\bar{\rho}=\langle\rho\rangle=\alpha \rho_{A}+(1-\alpha) \rho_{B}
$$

is the average density and

$$
\hat{K}=\left\langle K^{-1}\right\rangle^{-1}=\left(\frac{\alpha}{K_{A}}+\frac{(1-\alpha)}{K_{B}}\right)^{-1}
$$

is the harmonic average of the bulk moduli. The velocity (2.4) reduces to (2.3) if $Z_{A}=Z_{B}$, but more generally (2.3) does not hold. In particular, if $c_{A}=c_{B} \equiv c$ but $Z_{A} \neq Z_{B}$, then $\bar{c}<c$, so that even though all waves propagate with speed $c$, the effective velocity observed will be smaller. This is because the wave is constantly reflecting at each interface, and thus the energy propagates more slowly than the local wave speed.

The layered linear medium can be modeled by a homogenized equation with a dispersion relation of the form

$$
\omega=\bar{c} \xi+d \xi^{3}+\cdots,
$$

where $\xi$ is the spatial wavenumber and $\omega$ the temporal frequency. The effective speed $\bar{c}$ and the dispersion coefficient $d$ were derived by Santosa and Symes [6] using Bloch wave expansions. These can also be determined from the more general homogenized equations derived in section 5 for the nonlinear case.

Figures 1 and 2 show a comparison of waves propagating in a homogeneous medium and two different layered media. In each case the initial data are $q(x, 0) \equiv 0$ for $x \geq 0$, and a wave is generated by motion of the left boundary,

$$
u(0, t)= \begin{cases}\bar{u}(1+\cos (\pi(t-10) / 10)) & \text { if } 0 \leq t \leq 20 \\ 0 & \text { if } t>20\end{cases}
$$

The left edge is pulled outward for $0<t<20$, generating a strain wave that propagates to the right. Since the equations are linear, the magnitude of the disturbance scales out and we take $\bar{u}=1$, but for the nonlinear case the magnitude will be important.

In the figures, the solution is shown at time $t=40$, and four different quantities are displayed in each case: the strain $\epsilon(x, t)$, the corresponding stress $\sigma(\epsilon(x, t), x)$, the 

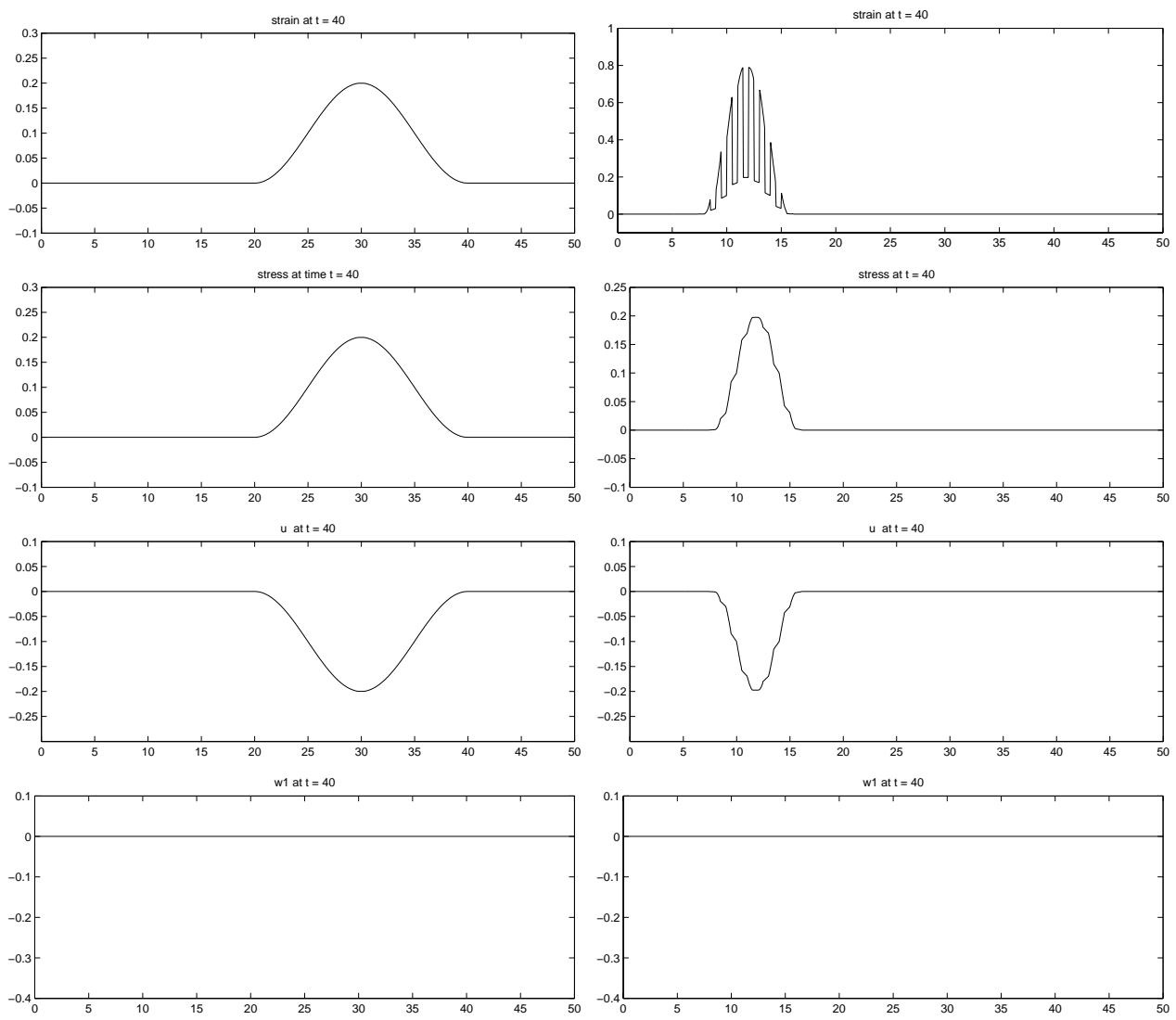

FIG. 1. The left column shows a strain wave propagating in a homogeneous medium with wave speed $c=1$. The right column shows a strain wave propagating in a layered medium with constant impedance. In each case four quantities are shown: strain, stress, velocity, and the characteristic variable $w^{1}(x, t)$, at time $t=40$.

velocity $u(x, t)$, and $w^{1}(x, t)$, where the characteristic variables $w^{1}$ and $w^{2}$ are defined by

$$
\begin{aligned}
& w^{1}(x, t)=\frac{1}{2 Z(x)}(Z(x) \epsilon(x, t)+\rho(x) u(x, t)), \\
& w^{2}(x, t)=\frac{1}{2 Z(x)}(Z(x) \epsilon(x, t)-\rho(x) u(x, t)) .
\end{aligned}
$$

These satisfy $w(x, t)=R^{-1}(x) q(x, t)$, where $R(x)$ is the matrix of right eigenvectors of the coefficient matrix $A(x)$ given in (2.2):

$$
R(x)=\left[\begin{array}{cc}
1 & 1 \\
Z(x) & -Z(x)
\end{array}\right], \quad R^{-1}(x)=\frac{1}{2 Z(x)}\left[\begin{array}{cc}
1 & Z(x) \\
1 & -Z(x)
\end{array}\right] .
$$

The quantities $w^{1}$ and $w^{2}$ give the magnitude of leftgoing and rightgoing waves, respectively.

The left column of Figure 1 shows results for a homogeneous medium with $\rho \equiv 1$, $K \equiv 1$. The wave moves at velocity $c=1$ and so lies between $x=20$ and $x=40$ at 

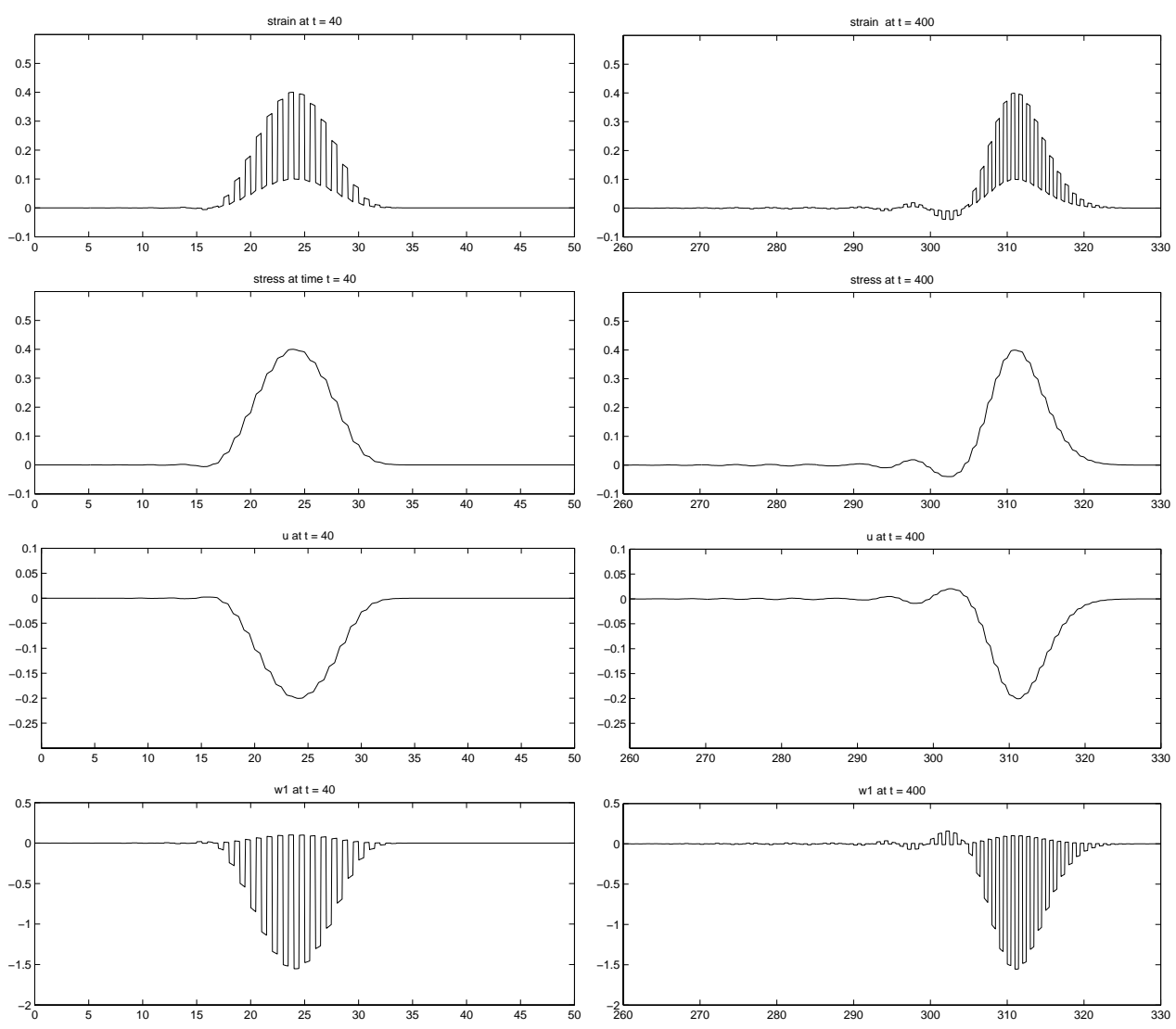

FIG. 2. A strain wave propagating in a layered medium with impedance mismatch. The left column shows the solution at time $t=40$, the right column at time $t=400$. In each case four quantities are shown: strain, stress, velocity, and the characteristic variable $w^{1}(x, t)$.

time $t=40$, with peak at $x=30$. Note that $w^{1}(x, t) \equiv 0$ since the wave is purely rightgoing.

The right column of Figure 1 shows a layered medium in which $\delta=1$ and

$$
\begin{array}{lllll}
\delta_{A}=0.5, & \rho_{A}=4, & K_{A}=0.25, & c_{A}=0.25, & Z_{A}=1, \\
\delta_{B}=0.5, & \rho_{B}=1, & K_{B}=1, & c_{B}=1, & Z_{B}=1 .
\end{array}
$$

In this case $Z_{A}=Z_{B}$, and so again the wave is purely rightgoing $\left(w^{1}(x, t) \equiv 0\right)$ and propagates with velocity $\bar{c}=2 / 5$ from (2.3). The peak of the disturbance is now observed at $x=30 \bar{c}=12$. Note that in this case the strain $\epsilon(x, t)$ is discontinuous at each layer interface. The B layers are more easily stretched than the A layers since $K_{B}>K_{A}$. The stress $\sigma(\epsilon(x, t), x)$ must be continuous, however, since the force acting on each side of the interface must be equal. This condition can be used to find the jump conditions on $\epsilon$. Similarly, the velocity $u(x, t)$ must be continuous everywhere, but the momentum $\rho(x) u(x, t)$ will be discontinuous at the interfaces where $\rho(x)$ has a jump discontinuity. 
The left column of Figure 2 shows a layered medium in which

$$
\begin{array}{lllll}
\delta_{A}=0.5, & \rho_{A}=4, & K_{A}=4, & c_{A}=1, & Z_{A}=4, \\
\delta_{B}=0.5, & \rho_{B}=1, & K_{B}=1, & c_{B}=1, & Z_{B}=1 .
\end{array}
$$

In this case $Z_{A} \neq Z_{B}$, and we have

$$
\bar{\rho}=\frac{5}{2}, \quad \hat{K}=\frac{8}{5}, \quad \bar{c}=\sqrt{\frac{\hat{K}}{\bar{\rho}}}=\frac{4}{5} .
$$

The peak of the disturbance is now located at $x=30 \bar{c}=24$. Note that in this case $w^{1}(x, t)$ is not identically zero, showing that the wave has a significant leftgoing component, although the envelope of $w^{1}$ propagates to the right at the effective velocity $\bar{c}$.

The right column of Figure 2 shows this same wave at a much later time, $t=400$, at which point the dispersive effect of the layered medium is apparent. The leading edge of the wave is still at approximately $\bar{c} t=320$, as expected, but trailing oscillations have appeared behind the wave due to the dispersion.

3. Waves in nonlinear media. We now consider the case of a nonlinear stressstrain relation $\sigma(\epsilon, x)$. In particular, we consider the exponential relation

$$
\sigma(\epsilon, x)=e^{K(x) \epsilon}-1,
$$

which will be related to the Toda lattice in section 4, and the simpler quadratic relation

$$
\sigma(\epsilon, x)=K(x) \epsilon+\beta K^{2}(x) \epsilon^{2},
$$

which approximates the exponential relation if $\beta=1 / 2$, and reduces to the linear case if $\beta=0$. For both of these nonlinear constitutive relations $\sigma_{\epsilon}(\epsilon, x)=K(x)+O(\epsilon) \rightarrow$ $K(x)$ as $\epsilon \rightarrow 0$, and so for very small amplitude waves the linear theory of the last section applies with bulk modulus $K(x)$.

The system of conservation laws (1.1) can now be written as

$$
q_{t}+f(q, x)_{x}=0
$$

or, for smooth solutions, as

$$
q_{t}+f_{q}(q, x) q_{x}=-f_{x}(q, x) .
$$

Note that we distinguish between $f(q, x)_{x}$, the total derivative of $f(q(x, t), x)$ with respect to $x$, and $f_{x}(q, x)$, the partial derivative of $f(q, x)$ with respect to the second variable. The Jacobian matrix for the system (3.4) is

$$
f_{q}(q, x)=\left[\begin{array}{cc}
0 & 1 / \rho(x) \\
\sigma_{\epsilon}(\epsilon, x) & 0
\end{array}\right],
$$

with eigenvalues $\lambda^{1}=-c$ and $\lambda^{2}=+c$, where the sound speed $c$ now depends on the strain as well as $x$,

$$
c(\epsilon, x)=\sqrt{\frac{\sigma_{\epsilon}(\epsilon, x)}{\rho(x)}} .
$$



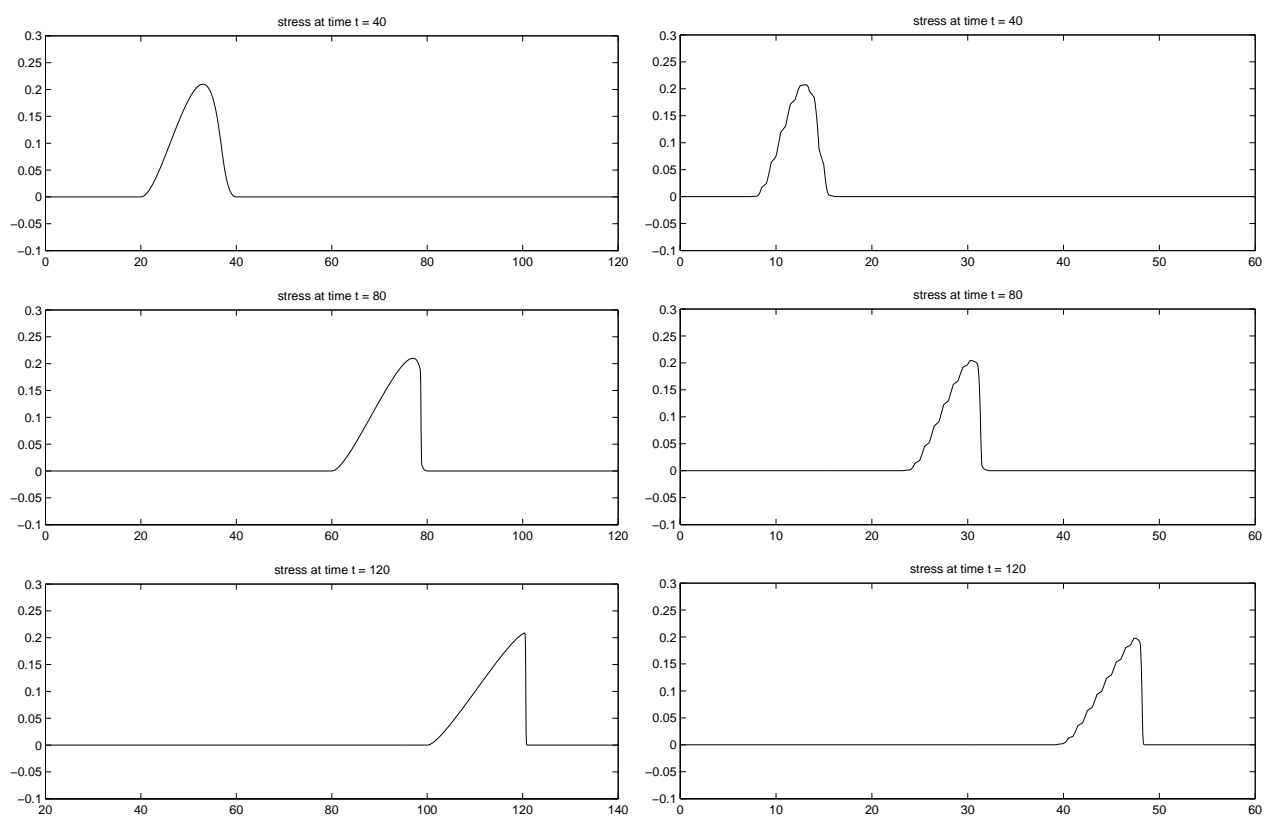

FIG. 3. The left column shows a wave propagating in a homogeneous nonlinear medium. Only the stress is shown at three different times, illustrating shock formation. The right column shows propagation in a layered nonlinear medium with constant linearized impedance.

The corresponding right eigenvectors of $f_{q}$ are

$$
r^{1}=\left[\begin{array}{c}
1 \\
Z(\epsilon, x)
\end{array}\right], \quad r^{2}=\left[\begin{array}{c}
1 \\
-Z(\epsilon, x)
\end{array}\right]
$$

where the impedance $Z(\epsilon, x)=\sqrt{\rho(x) \sigma_{\epsilon}(\epsilon, x)}$ now also depends on $\epsilon$. For either constitutive model (3.1) or (3.2) we have $c(\epsilon, x) \rightarrow \sqrt{K(x) / \rho(x)}$ and $Z(\epsilon, x) \rightarrow$ $\sqrt{\rho(x) K(x)}$ as $\epsilon \rightarrow 0$, which are the linearized sound speed and impedance.

Figures 3 and 4 show computed results for three cases analogous to those shown for the linear problem in the previous section, but now using the exponential relation (3.1) and $\bar{u}=0.2$ in the boundary data (2.8). Here we display only one quantity, the stress $\sigma(\epsilon(x, t), x)$, but plot the solution at several different times to show the evolution.

The left column of Figure 3 shows nonlinear propagation in a homogeneous medium, with $\rho \equiv 1, K \equiv 1$. The cosine-shaped wave generated by the boundary condition (2.8) steepens into a shock followed by a rarefaction wave, as expected from standard nonlinear conservation law theory.

The right column of Figure 3 shows a layered medium with

$$
\begin{array}{lll}
\delta_{A}=0.5, & \rho_{A}=4, & K_{A}=0.25, \\
\delta_{B}=0.5, & \rho_{B}=1, & K_{B}=1 .
\end{array}
$$

In this case the linearized impedances are matched since $\rho_{A} K_{A}=\rho_{B} K_{B}$, and again the rightgoing wave appears to steepen into a shock followed by a rarefaction wave. However, the full nonlinear impedance does not remain matched, and some reflection occurs at interfaces. This shock wave is not a sharp discontinuity but remains smeared 

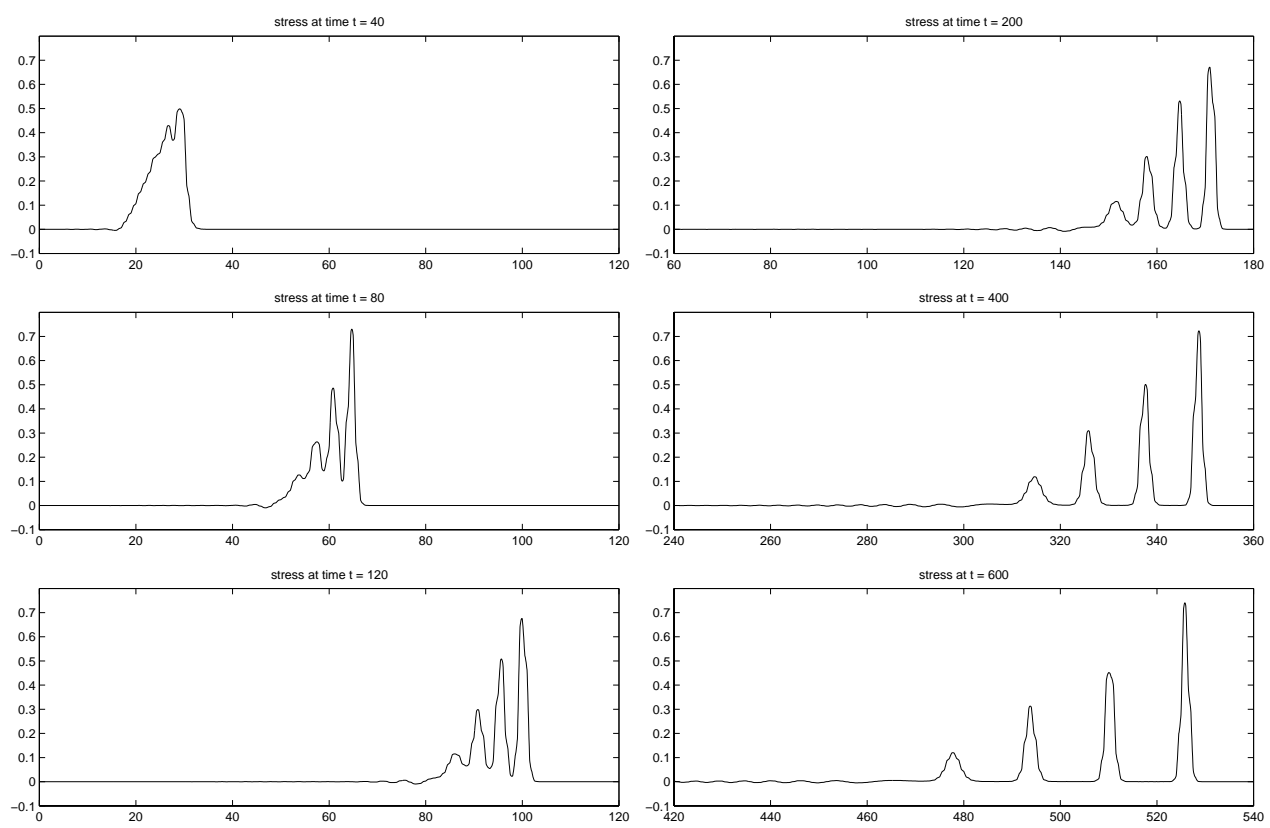

FIG. 4. Propagation in a layered nonlinear medium with impedance mismatch at the interfaces. The left column shows the stress at three times, and the right column at three later times, illustrating breakup into a train of solitary waves.

over a few layers. In this case the layering gives rise to an effective viscous equation. (In this case $C_{13}$ is nonzero in (5.17) below, while the other $C$ coefficients are all zero.)

Figure 4 shows a more interesting case that is the primary object of study in this paper. Here the material parameters are

$$
\begin{array}{lll}
\delta_{A}=0.5, & \rho_{A}=4, & K_{A}=4, \\
\delta_{B}=0.5, & \rho_{B}=1, & K_{B}=1 .
\end{array}
$$

Now the linearized impedance is not matched, and large-scale reflections at each interface lead to dispersive behavior. This dispersion, coupled with the nonlinearity, results in the existence of solitary waves. In Figure 4 the same boundary motion (2.8) is applied as before. The resulting pulse, which is long compared to the layer width, initially starts to steepen as if a shock were forming. But then oscillations develop and the pulse ultimately breaks up into a train of solitary waves. Similar behavior is seen with nonlinear dispersive equations such as the KdV equation that are known to have soliton solutions.

Figure 5 shows both the stress and the strain in the first two solitary waves at time $t=600$. Since it is not clear whether these solitary waves are formally solitons, we will refer to them as stegotons for shorthand, coming from the Greek root "stego-," meaning roof or ridge, and suggested by the rough resemblance of these strain waves in a layered medium to the back of a stegosaurus.

Note that each stegoton has a width of about ten layers, a fact that is independent of the periodicity $\delta$ used. If $\delta$ is made smaller, then the stegotons scale with $\delta$ but remain about ten layers wide. This is expected from the form of the equations. If $\tilde{q}(x, t)$ is a solution to (3.3) for $\delta=1$, then $q(x, t)=\tilde{q}(x / \delta, t / \delta)$ is a solution for arbitrary $\delta$ (with $\alpha$ fixed). The width in layers does vary slightly with amplitude, as 

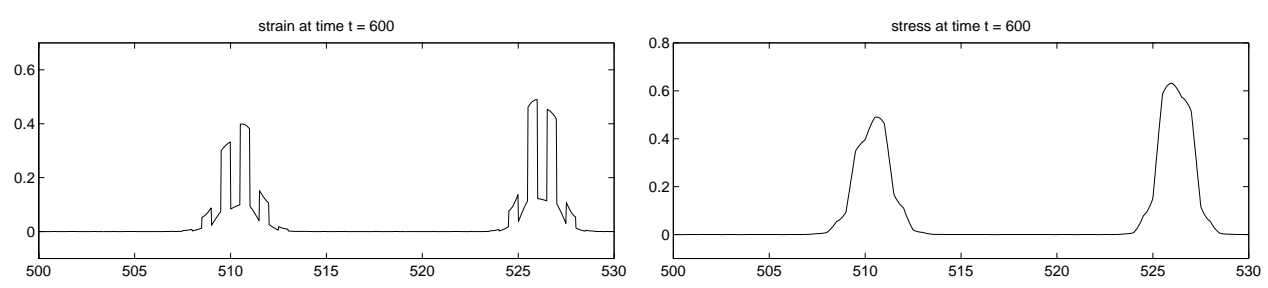

FIG. 5. Close-up view of the first two solitary waves from Figure 4 at time $t=600$, showing both the strain and the stress.
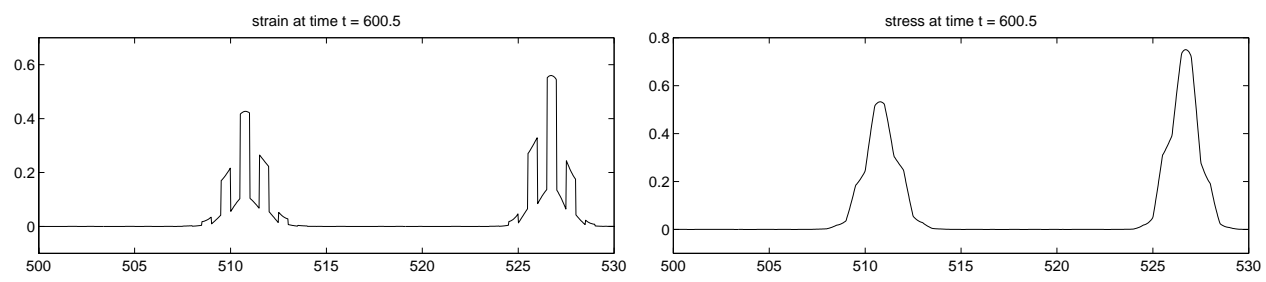

FIG. 6. Close-up view of the two solitary waves from Figure 5 at a slightly later time $t=600.5$.

(a)

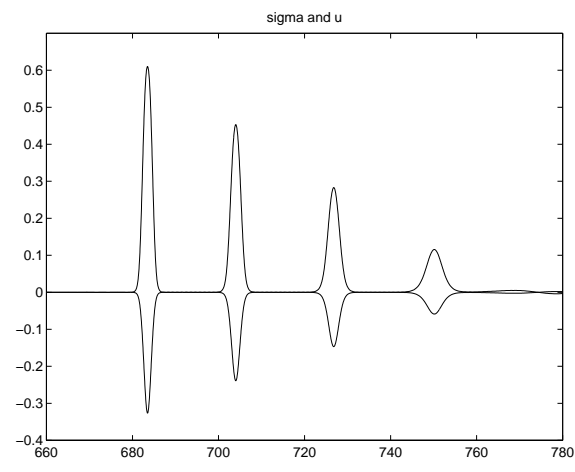

(b)

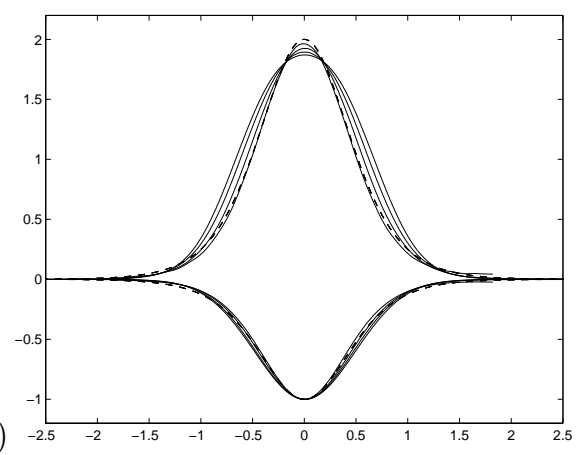

FIG. 7. (a) A time trace of the stress $\sigma\left(x_{0}, t\right) \geq 0$ and velocity $u\left(x_{0}, t\right) \leq 0$ at a fixed point $x_{0}$ as four stegotons pass by. (b) The same four waves replotted as functions of $\tau$ with the scaling described in the text. The dashed lines are $-\operatorname{sech}^{2}(b \tau)$ and $2 \operatorname{sech}^{2}(b \tau)$.

does the speed of the solitary wave. The taller stegotons are thinner and travel faster than shorter ones, similar to the behavior of KdV solitons, for example.

Stegotons do not translate with a fixed wave shape, as illustrated in Figure 6, where the two stegotons from Figure 5 are shown at a slightly later time, $t=600.5$. For this reason it is difficult to carefully investigate the scaling properties of stegotons by studying their appearance as functions of $x$ for fixed $t$. On the other hand, if we pick a physical location $x$ and observe the solution as a function of $t$ as a stegoton passes by, then all components of the solution vary smoothly in time. By observing the waves in this manner it is easy to determine the scaling relation between amplitude, speed, and width. Figure 7(a) shows the stress $\sigma\left(x_{0}, t\right)$ as a function of $t$ at $x_{0}=600.25$, a location that is in the center of an A-layer. This shows four distinct solitary waves passing by, followed by some trailing noise. Here we have also plotted the velocity $u\left(x_{0}, t\right)$, which is negative in each wave.

Figure 7(b) shows plots of the four leading solitary waves from Figure 7(a) after 


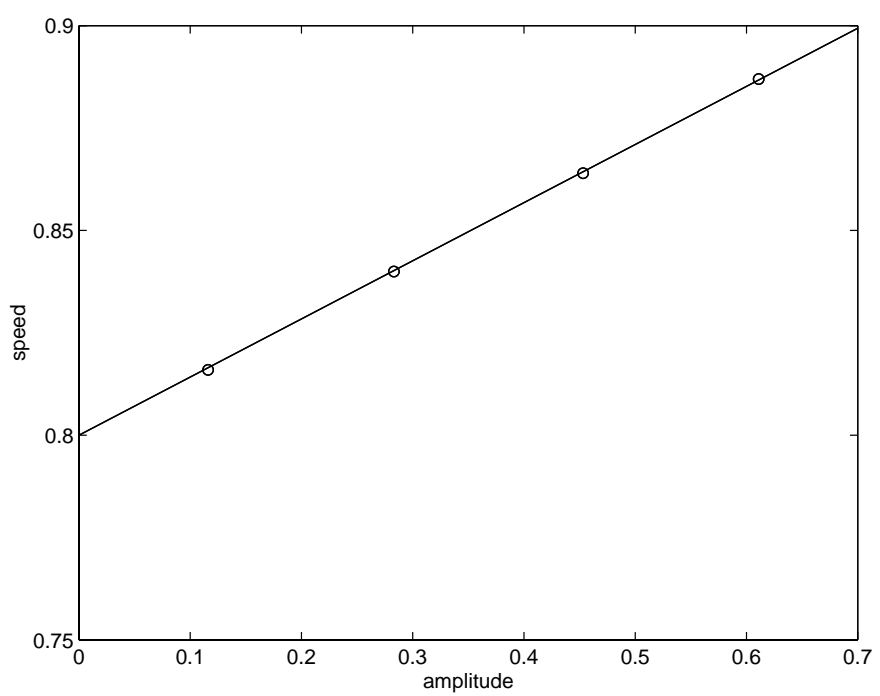

FIG. 8. The observed speed of a solitary wave plotted as a function of amplitude, for the four waves shown in Figure 7.

shifting them to a common location, rescaling each by its amplitude $a$ as measured from the velocity, $a=\max |u|$, and rescaling the width by $\sqrt{a}$. Hence we plot

$$
\frac{1}{a} \sigma\left(x_{0}, \tau\right) \quad \text { and } \quad \frac{1}{a} u\left(x_{0}, \tau\right)
$$

as functions of $\tau$, where $\tau=\sqrt{a}\left(t-t_{m}\right)$ and $t_{m}$ is the time at which the velocity reaches its peak value $-a$. The velocity plots lie nearly on top of one another, suggesting that at $x=x_{0}$ a stegoton of amplitude $a$ has velocity of the form

$$
u\left(x_{0}, t\right)=a U\left(\sqrt{a}\left(t-t_{m}\right)\right)
$$

for some function $U(\tau)$. The dashed lines in Figure $7(\mathrm{~b})$ show the functions $-\operatorname{sech}^{2}(b \tau)$ and $2 \operatorname{sech}^{2}(b \tau)$ for $b=1.7$. This shows that the stegoton has roughly, though not exactly, the sech ${ }^{2}$ shape seen for many solitons.

The stress does not scale quite as nicely as the velocity, and in particular the stress is not simply a scalar multiple of the velocity. It is nearly so, however, for the stress curve that appears tallest in Figure 7(b). This actually corresponds to the shortest stegoton in the original time trace of Figure 7(a). For this small amplitude wave we observe $\sigma \approx-2 u$. This is consistent with the fact that a linearized homogeneous medium with impedance $Z$ would have $\sigma=-Z u$, and the layered medium we are using has an effective impedance in the linearized case that is $(\hat{K} \bar{\rho})^{1 / 2}=2$.

By observing when the peak appears in the time history at different points $x$ (each in the center of an A-layer), we can estimate the velocity of each wave. The observed velocity is plotted against the amplitude in Figure 8 for each of these four stegotons. They lie almost exactly on the line

$$
v=0.8+0.142 a \text {. }
$$

Recall that $\bar{c}=0.8$ is the effective velocity of the linearized medium from (2.13), which is the velocity we expect to observe for very small amplitude waves. The velocity appears to increase linearly with amplitude for nonlinear waves. 

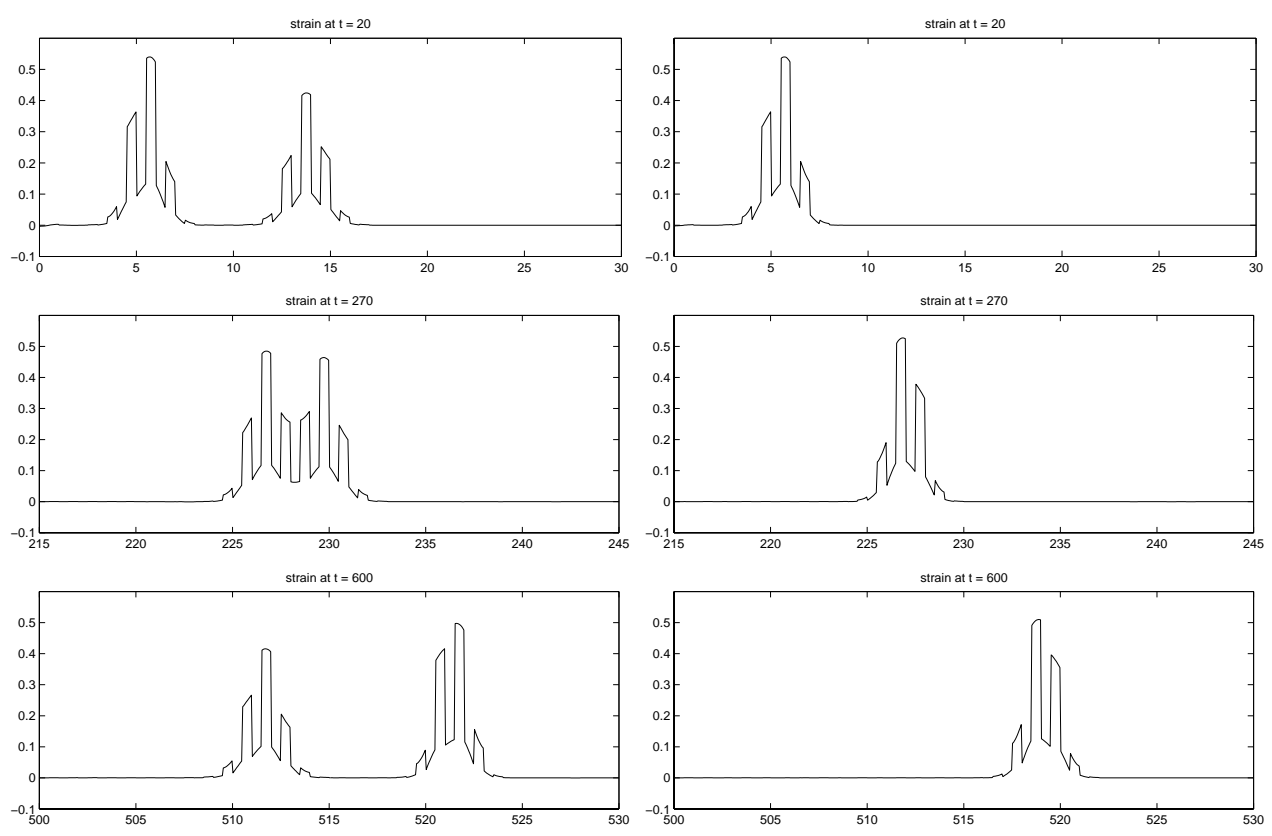

FIG. 9. The collision of two stegotons at three different times is shown in the left column. For comparison, the right column shows results at the same times for a single stegoton.

These results are for the case in which $x_{0}$ is in the center of an A-layer. If we fix $x_{0}$ at a different spot, then the scaling is the same but the amplitude $a$ and wave shape $U(\tau)$ are different. Indeed, the amplitude varies significantly at different points, even within the same layer. A more complete characterization of these waves is still under development.

By recording $u\left(x_{0}, t\right)$ at a location $x_{0}$ that is at the left edge of an A-layer, and then selecting the part of this time history that captures a single wave passing by, we obtain data that can be used to replace (2.8) as boundary data for generating a single stegoton. By rescaling the amplitude and width of this data appropriately using the scaling determined above, we can also generate stegotons of arbitrary amplitude $a$. We have verified that these also propagate as solitary waves, at least if the amplitude is not too large.

We can also generate a short stegoton at the boundary followed by a taller stegoton that travels faster and eventually overtakes the first. The left column of Figure 9 shows the results of this experiment. We observe that the two waves interact in a manner analogous to classical solitons: the waves appear to exchange identity, and the wave in front grows and accelerates. After the waves separate, each again has the form of a solitary stegoton, though shifted in location from where they would be without interaction. For comparison, the right column of Figure 9 shows the propagation of the larger stegoton alone, without the presence of the smaller one.

4. Relation to the Toda lattice. The Toda lattice is a discrete lattice of particles having mass $m$ connected by nonlinear springs with a restoring force that depends exponentially on the distance stretched. Let $X_{j}(t)$ be the location of the $j$ th particle at time $t$, and assume that the unstretched configuration has $X_{j}=j \Delta x$. The velocity of this particle is denoted by $U_{j}(t)$. The spring connecting particle $j$ to $j+1$ 
has strain

$$
\epsilon_{j+1 / 2}(t)=\frac{X_{j+1}(t)-X_{j}(t)}{\Delta x}-1
$$

and exerts a restoring force $\sigma\left(\epsilon_{j+1 / 2}(t)\right)$, where

$$
\sigma(\epsilon)=e^{K \epsilon}-1 .
$$

Since $X_{j}^{\prime}(t)=U_{j}(t)$, differentiating (4.1) yields

$$
\epsilon_{j+1 / 2}^{\prime}(t)=\frac{U_{j+1}(t)-U_{j}(t)}{\Delta x} .
$$

The Toda lattice is modeled by a system of ODEs consisting of this equation along with the dynamic equation

$$
m U_{j}^{\prime}(t)=\sigma\left(\epsilon_{j+1 / 2}(t)\right)-\sigma\left(\epsilon_{j-1 / 2}(t)\right) .
$$

If we rewrite $m=\rho \Delta x$, then the system of equations becomes

$$
\begin{aligned}
\epsilon_{j+1 / 2}^{\prime}(t) & =\frac{U_{j+1}(t)-U_{j}(t)}{\Delta x}, \\
\rho U_{j}^{\prime}(t) & =\frac{\sigma\left(\epsilon_{j+1 / 2}(t)\right)-\sigma\left(\epsilon_{j-1 / 2}(t)\right)}{\Delta x} .
\end{aligned}
$$

Note that this can be viewed as a finite-difference discretization of the elastic equation (1.1). Centered differences on a staggered grid are used to approximate each $x$ derivative in (1.1). The classical theory of finite-difference methods thus leads us to expect dispersive behavior, and a "modified equation" analysis of this system would show that the discrete equations can be approximated by a dispersive nonlinear system of PDEs. With this combination of nonlinearity and dispersion, solitary waves can arise. Toda showed that with exponential springs the discrete system is completely integrable and discrete soliton solutions exist; see [7], [8].

To relate the Toda lattice to a layered medium, it may be tempting to introduce two different types of springs with constitutive relations $\sigma_{A}(\epsilon)$ and $\sigma_{B}(\epsilon)$ and have these alternate in the discrete lattice. However, this would introduce a second form of dispersion and result in a doubly dispersive system.

Since the original Toda lattice already has soliton solutions, we wish to relate the layered medium directly to the lattice. This is easily done by realizing that the Toda lattice does in fact consist of alternating layers, since particles alternate with springs. The corresponding layered medium is one in which thin "particle layers" with finite mass and infinitesimal compressibility alternate with thicker "spring layers" having infinitesimal mass and finite compressibility, i.e.,

$$
\begin{array}{lll}
\delta_{A} \ll 1, & \rho_{A}=O\left(\frac{1}{\delta_{A}}\right), & K_{A} \gg 1, \\
\delta_{B}=1-\delta_{A}, & \rho_{B} \ll 1, & K_{B}=O(1) .
\end{array}
$$

See Figure 10 for an illustration of this correspondence. (In fact, one observes close correspondence even when $\delta_{A}$ is not small relative to $\delta_{B}$.) 

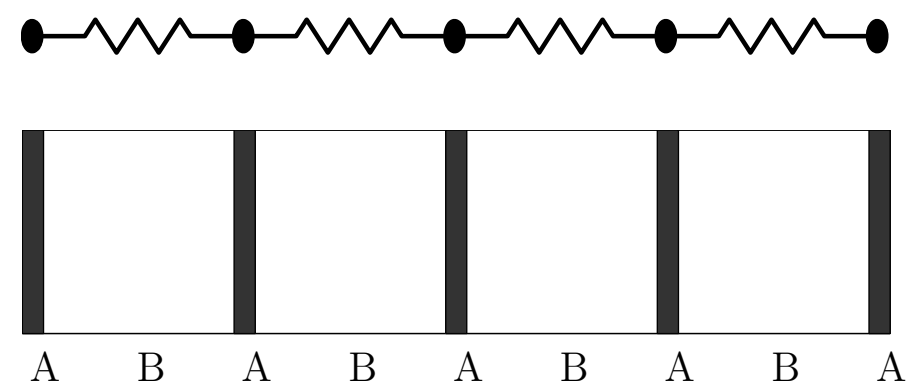

FIG. 10. The Toda lattice and a roughly equivalent layered medium.
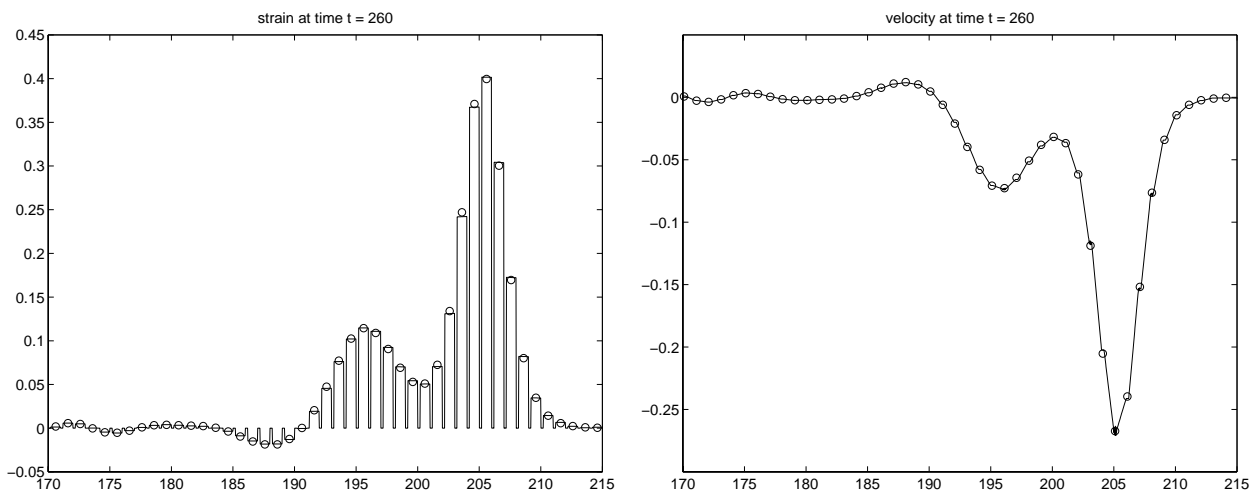

FIG. 11. Comparison of the solution to the discrete Toda lattice (circles) with the finite-volume solution to the layered medium equations (solid line). The strain and velocity are shown.

Figure 11 shows a sample calculation obtained by solving the layered media equations using

$$
\begin{aligned}
& \delta_{A}=0.2, \quad \rho_{A}=5, \quad K_{A}=2000 \\
& \delta_{B}=0.8, \quad \rho_{B}=0.005, \quad K_{B}=0.5 .
\end{aligned}
$$

In the particle layers, which exhibit little strain, the constitutive relation is taken to be linear with $\sigma_{A}(\epsilon)=K_{A} \epsilon$. The wave speed is then constant in these layers, $c_{A}=20$, which is convenient numerically since by taking $\Delta t=\Delta x / 20$ (so the Courant number is exactly 1) an accurate solution is computed even when there are very few points in these thin layers. The spring layers have the exponential relation $\sigma_{B}(\epsilon)=e^{K_{B} \epsilon}-1$, with a wave speed that approaches $c_{B}=\sqrt{K_{B} / \rho_{B}}=10$ for small $\epsilon$ and remains below 20 so that the calculations are stable.

The parameters (4.6) lead to an effective wave speed for the linearized response (small $\epsilon$ ) of

$$
\bar{c}=\sqrt{\frac{\hat{K}}{\bar{\rho}}}=\sqrt{\frac{0.625}{1.004}} \approx 0.789,
$$

and solitary waves move with a speed that is less than 1 . To have a close connection with the Toda lattice, it is important that the wave speeds $c_{A}$ and $c_{B}$ are much larger than this homogenized wave speed. This means that small amplitude waves bounce 
back and forth within each layer on a much faster time scale than the observed wave motion, leading to a local equilibration within each layer. As a result, the stress observed in Figure 11 at any time is roughly constant in each thick spring layer, and varies rapidly, but essentially linearly, in the thin particle layers. The velocity $u$, on the other hand, is roughly constant in each particle layer, since the particle moves as a rigid unit, and varies linearly across a spring layer. Only under these conditions can we hope to model the continuum material by a set of discrete springs and masses, where each spring has a single stress associated with it and is assumed to compress and expand uniformly.

Figure 11 shows that there is in fact good agreement between the layered medium and the Toda lattice in this case. The parameters for the Toda lattice used here are $m=1.004$ for the particle mass and $K=0.5$ in the stress-strain relation. The mass is the average density from the layered medium, while the value of $K$ is taken to be $K_{B}$, the corresponding parameter from the spring layers. Note that, for the layered medium solution (solid line), the strain is nearly zero in each thin particle layer and nearly constant throughout each spring layer. The circles show the discrete solution computed by solving the ODEs of the Toda lattice. These results are shown at a time when the initial pulse is just breaking up into solitary waves. At later times both solutions break up into similar trains of solitary waves.

Because of the correspondence in Figure 10, it is not surprising that the layered medium exhibits solitary waves in this special case. What is more interesting is the fact that it continues to exhibit solitary wave behavior even for situations that are far from this limit, as was exhibited in section 3. In the next section we derive homogenized equations for the general case that may help to shed some light on this.

5. Homogenized equations. In this section we derive homogenized equations that describe the effective behavior of the layered media (both linear and nonlinear) studied in the previous sections. Since the strain and momentum have discontinuities at each interface and cannot be approximated directly by continuous functions, we start by rewriting the equations in terms of the stress and velocity, which are continuous. Equation (1.1b), $\rho(x) u_{t}-\sigma_{x}=0$, is one equation of this system. We must use (1.1a) to derive an equation for $\sigma_{t}$. To do so, we use

$$
\sigma_{t}=\sigma_{\epsilon}(\epsilon, x) \epsilon_{t}=\sigma_{\epsilon}(\epsilon, x) u_{x}
$$

and will assume that the constitutive relation $\sigma(\epsilon, x)$ is such that we can solve for $\sigma_{\epsilon}(\epsilon, x)$ as a function of $\sigma$ and $x$ in the form

$$
\sigma_{\epsilon}(\epsilon, x)=K(x) G(\sigma) .
$$

In particular, for the constitutive equations used in this paper we have

$$
\begin{array}{ll}
\sigma(\epsilon, x)=K(x) \epsilon & \Longrightarrow G(\sigma)=1, \\
\sigma(\epsilon, x)=\exp (K(x) \epsilon)-1 & \Longrightarrow G(\sigma)=1+\sigma, \\
\sigma(\epsilon, x)=K(x) \epsilon+\beta K^{2}(x) \epsilon^{2} \quad \Longrightarrow \quad G(\sigma)=1+2 \beta \sigma-2 \beta^{2} \sigma^{2}+O\left(\sigma^{3}\right) .
\end{array}
$$

Then the system (1.1) can be rewritten as

$$
\begin{aligned}
\sigma_{t}-K(x) G(\sigma) u_{x} & =0 \\
\rho(x) u_{t}-\sigma_{x} & =0 .
\end{aligned}
$$

This nonlinear system is not in conservation form but is still valid since we are not interested in shock waves. The leading order terms in the homogenized equation are 
easy to derive by rewriting (5.3) as

$$
\begin{aligned}
K^{-1}(x) \sigma_{t}-G(\sigma) u_{x} & =0 \\
\rho(x) u_{t}-\sigma_{x} & =0 .
\end{aligned}
$$

The functions $K(x)$ and $\rho(x)$ vary on a much faster scale than $\sigma$ and $u$, and thus averaging these equations over a period leads to

$$
\begin{aligned}
\left\langle K^{-1}(x)\right\rangle \sigma_{t}-G(\sigma) u_{x} & \approx 0 \\
\langle\rho(x)\rangle u_{t}-\sigma_{x} & \approx 0
\end{aligned}
$$

This gives a homogenized system that again has the form (5.3) but with $\rho(x)$ and $K(x)$ replaced by the average and harmonic average, respectively. This gives the expected effective velocity in the linear case, but is lacking the crucial dispersive terms needed to explain the solitary waves.

To obtain a more accurate description of the homogenized equations, we use a multiple scale homogenization technique, following [3]. We begin by defining a fast spatial variable $x^{*}=x / \delta$, where $\delta$ is the period of the medium, as before, and writing the bulk modulus and density as functions of $x^{*}: K=K\left(x^{*}\right)$ and $\rho=\rho\left(x^{*}\right)$. (This is a slight abuse of notation.) We now adopt the formalism that $\bar{x}=x$ and $x^{*}$ are independent variables by assuming $\delta \ll 1$. As a result, spatial derivatives in (5.3) must be transformed according to

$$
\frac{\partial}{\partial x}=\frac{\partial}{\partial \bar{x}}+\frac{1}{\delta} \frac{\partial}{\partial x^{*}}
$$

(We introduce $x=\bar{x}$ in this step to clearly delineate the original spatial scale $x$ and the new multiple scales $\bar{x}$ and $x^{*}$, but we will simply use $x$ from now on.) System (5.3) becomes

$$
\begin{aligned}
\sigma_{t}-K\left(x^{*}\right) G(\sigma)\left[u_{x}+\delta^{-1} u_{x^{*}}\right] & =0 \\
\rho\left(x^{*}\right) u_{t}-\left[\sigma_{x}+\delta^{-1} \sigma_{x^{*}}\right] & =0 .
\end{aligned}
$$

Using the convention that all underlined quantities are independent of the fast variable $x^{*}$, we insert the asymptotic expansions

$$
\begin{aligned}
& u(x, t ; \delta)=\underline{u}^{(0)}(x, t)+\delta u^{(1)}\left(x, x^{*}, t\right)+\delta^{2} u^{(2)}\left(x, x^{*}, t\right)+O\left(\delta^{3}\right), \\
& \sigma(x, t ; \delta)=\underline{\sigma}^{(0)}(x, t)+\delta \sigma^{(1)}\left(x, x^{*}, t\right)+\delta^{2} \sigma^{(2)}\left(x, x^{*}, t\right)+O\left(\delta^{3}\right)
\end{aligned}
$$

into (5.6) and collect terms by their powers of $\delta$. During this process, the function $G(\sigma)$ must be expanded as

$$
\begin{aligned}
G(\sigma)=G\left(\underline{\sigma}^{(0)}+\delta \sigma^{(1)}+\delta^{2} \sigma^{(2)}+\cdots\right) \\
=G\left(\underline{\sigma}^{(0)}\right)+G^{\prime}\left(\underline{\sigma}^{(0)}\right)\left(\delta \sigma^{(1)}+\delta^{2} \sigma^{(2)}+\cdots\right) \\
\quad+\frac{1}{2} G^{\prime \prime}\left(\underline{\sigma}^{(0)}\right)\left(\delta \sigma^{(1)}+\delta^{2} \sigma^{(2)}+\cdots\right)^{2}+\cdots .
\end{aligned}
$$

Once the proper choices for scales and asymptotic expansions have been made, obtaining homogenized equations becomes a purely mechanical, although algebraically intensive, procedure. For the system of equations that is proportional to $\delta^{n}$, we solve for $u_{x^{*}}^{(n+1)}$ and $\sigma_{x^{*}}^{(n+1)}$ and identify any terms that are independent of $x^{*}$. These terms 
must be set to zero; otherwise, when $u_{x^{*}}^{(n+1)}$ and $\sigma_{x^{*}}^{(n+1)}$ are integrated with respect to $x^{*}$, secular terms will arise. The terms that are set to zero give rise to the homogenized equations.

To identify terms that are independent of $x^{*}$, we introduce the following linear operators:

$$
\begin{aligned}
\left\langle a\left(x^{*}\right)\right\rangle & =\int_{0}^{1} a\left(x^{*}\right) d x^{*}, \\
\{a\}\left(x^{*}\right) & =a\left(x^{*}\right)-\left\langle a\left(x^{*}\right)\right\rangle, \\
\llbracket a \rrbracket\left(x^{*}\right) & =\int_{s}^{x^{*}}\{a\}(\xi) d \xi, \quad \text { where } s \text { is chosen such that }\left\langle\llbracket a \rrbracket\left(x^{*}\right)\right\rangle=0 .
\end{aligned}
$$

When using the normal spatial scale, $\delta$ is the period of the medium, but when using the fast spatial scale $x^{*}=x / \delta$, the period of the functions $K\left(x^{*}\right)$ and $\rho\left(x^{*}\right)$ is 1 . Therefore, the averaging operator $\left\langle K\left(x^{*}\right)\right\rangle$, defined above, gives the average value of $K\left(x^{*}\right)$. (A similar averaging operator can be defined for nonperiodic functions.) The $\{\cdot\}$ operator generates the fluctuating part of a function: the part of the function that has zero average. The $\llbracket \cdot \rrbracket$ operator gives the integral of the fluctuating part of a function, where the constant of integration is chosen such that the average of the integral of the fluctuating part is zero. As opposed to $\langle\cdot\rangle$, both $\{\cdot\}$ and $\llbracket \cdot \rrbracket$ return functions of $x^{*}$. Some useful properties of these operators are derived in [11].

In the case of piecewise constant functions (1.2), $\left\langle\rho\left(x^{*}\right)\right\rangle=\bar{\rho}=\alpha \rho_{A}+(1-\alpha) \rho_{B}$,

$$
\{\rho\}\left(x^{*}\right)= \begin{cases}(1-\alpha)\left(\rho_{A}-\rho_{B}\right) & \text { if } j<x^{*}<(j+\alpha) \text { for some integer } j, \\ \alpha\left(\rho_{B}-\rho_{A}\right) & \text { otherwise }\end{cases}
$$

and

$$
\llbracket \rho \rrbracket\left(x^{*}\right)= \begin{cases}(1-\alpha)\left(\rho_{A}-\rho_{B}\right)\left(x^{*}-\frac{\alpha}{2}\right) & \text { if } j<x^{*}<(j+\alpha) \text { for some integer } j, \\ \alpha\left(\rho_{B}-\rho_{A}\right)\left(x^{*}-\frac{1+\alpha}{2}\right) & \text { otherwise. }\end{cases}
$$

Now we illustrate how the first few terms of the homogenized equations are derived. The leading order equations are

$$
\begin{aligned}
\underline{\sigma}_{t}^{(0)}-K\left(x^{*}\right) G\left(\underline{\sigma}^{(0)}\right)\left(\underline{u}_{x}^{(0)}+u_{x^{*}}^{(1)}\right) & =0, \\
\rho\left(x^{*}\right) \underline{u}_{t}^{(0)}-\underline{\sigma}_{x}^{(0)}-\sigma_{x^{*}}^{(1)} & =0 .
\end{aligned}
$$

We solve for $u_{x^{*}}^{(1)}$ and $\sigma_{x^{*}}^{(1)}$ to obtain

$$
\begin{aligned}
u_{x^{*}}^{(1)} & =\frac{\underline{\sigma}_{t}^{(0)}}{K\left(x^{*}\right) G\left(\underline{\sigma}^{(0)}\right)}-\underline{u}_{x}^{(0)}, \\
\sigma_{x^{*}}^{(1)} & =\rho\left(x^{*}\right) \underline{u}_{t}^{(0)}-\underline{\sigma}_{x}^{(0)} .
\end{aligned}
$$

Before integrating with respect to $x^{*}$, we must first remove $x^{*}$-independent terms from the right-hand sides of (5.8). To do this, we apply $\langle\cdot\rangle$ to the right-hand sides of (5.8) and set the result to zero:

$$
\begin{aligned}
& 0=\left\langle K^{-1}\right\rangle \underline{\sigma}_{t}^{(0)}-G\left(\underline{\sigma}^{(0)}\right) \underline{u}_{x}^{(0)}, \\
& 0=\langle\rho\rangle \underline{u}_{t}^{(0)}-\underline{\sigma}_{x}^{(0)} .
\end{aligned}
$$


We can then integrate the remaining terms in (5.8) to get

$$
\begin{aligned}
& u^{(1)}=\llbracket K^{-1} \rrbracket \frac{\underline{\sigma}_{t}^{(0)}}{G\left(\underline{\sigma}^{(0)}\right)}+\underline{u}^{(1)}, \\
& \sigma^{(1)}=\llbracket \rho \rrbracket \underline{u}_{t}^{(0)}+\underline{\sigma}^{(1)},
\end{aligned}
$$

where $\underline{u}^{(1)}$ and $\underline{\sigma}^{(1)}$ are "constants" of integration in terms of $x^{*}$ but vary with $x$ and $t$.

The $O(\delta)$ equations are

$$
\begin{array}{r}
\sigma_{t}^{(1)}-K\left(x^{*}\right) G\left(\underline{\sigma}^{(0)}\right)\left(u_{x}^{(1)}+u_{x^{*}}^{(2)}\right)-K\left(x^{*}\right) G^{\prime}\left(\underline{\sigma}^{(0)}\right) \sigma^{(1)}\left(\underline{u}_{x}^{(0)}+u_{x^{*}}^{(1)}\right)=0, \\
\rho\left(x^{*}\right) u_{t}^{(1)}-\sigma_{x}^{(1)}-\sigma_{x^{*}}^{(2)}=0 .
\end{array}
$$

We substitute in (5.7a) and (5.10) and solve for $u_{x^{*}}^{(2)}$ and $\sigma_{x^{*}}^{(2)}$ to obtain

$$
\begin{gathered}
u_{x^{*}}^{(2)}=\frac{\llbracket \rho \rrbracket \underline{u}_{t t}^{(0)}+\underline{\sigma}_{t}^{(1)}}{K\left(x^{*}\right) G\left(\underline{\sigma}^{(0)}\right)}-\llbracket K^{-1} \rrbracket \frac{G\left(\underline{\sigma}^{(0)}\right) \underline{\sigma}_{x t}^{(0)}-\underline{\sigma}_{t}^{(0)} \underline{\sigma}_{x}^{(0)} G^{\prime}\left(\underline{\sigma}^{(0)}\right)}{G\left(\underline{\sigma}^{(0)}\right)^{2}}-\underline{u}_{x}^{(1)} \\
\left.-\frac{G^{\prime}\left(\underline{\sigma}^{(0)}\right) \underline{\sigma}_{t}^{(0)}}{K\left(x^{*}\right) G\left(\underline{\sigma}^{(0)}\right)^{2}} \llbracket \llbracket \rho \rrbracket \underline{u}_{t}^{(0)}+\underline{\sigma}^{(1)}\right],
\end{gathered}
$$

$$
\sigma_{x^{*}}^{(2)}=\rho\left(x^{*}\right)\left[\llbracket K^{-1} \rrbracket \frac{G\left(\underline{\sigma}^{(0)}\right) \underline{\sigma}_{t t}^{(0)}-\left(\underline{\sigma}_{t}^{(0)}\right)^{2} G^{\prime}\left(\underline{\sigma}^{(0)}\right)}{G\left(\underline{\sigma}^{(0)}\right)^{2}}+\underline{u}_{t}^{(1)}\right]-\llbracket \rho \rrbracket \underline{u}_{x t}^{(0)}-\underline{\sigma}_{x}^{(1)} .
$$

We remove $x^{*}$-independent terms by setting

$$
\begin{gathered}
0=\left\langle\llbracket \rho \rrbracket K^{-1}\right\rangle \underline{u}_{t t}^{(0)}+\left\langle K^{-1}\right\rangle \underline{\sigma}_{t}^{(1)}-G\left(\underline{\sigma}^{(0)}\right) \underline{u}_{x}^{(1)}-\left\langle\llbracket \rho \rrbracket K^{-1}\right\rangle \frac{\underline{u}_{t}^{(0)} \underline{\sigma}_{t}^{(0)} G^{\prime}\left(\underline{\sigma}^{(0)}\right)}{G\left(\underline{\sigma}^{(0)}\right)} \\
-\left\langle K^{-1}\right\rangle \frac{\underline{\sigma}^{(1)} \underline{\sigma}_{t}^{(0)} G^{\prime}\left(\underline{\sigma}^{(0)}\right)}{G\left(\underline{\sigma}^{(0)}\right)}
\end{gathered}
$$

$$
0=\left\langle\rho \llbracket K^{-1} \rrbracket\right\rangle \frac{G\left(\underline{\sigma}^{(0)}\right) \underline{\sigma}_{t t}^{(0)}-\left(\underline{\sigma}_{t}^{(0)}\right)^{2} G^{\prime}\left(\underline{\sigma}^{(0)}\right)}{G\left(\underline{\sigma}^{(0)}\right)^{2}}+\langle\rho\rangle \underline{u}_{t}^{(1)}-\underline{\sigma}_{x}^{(1)} .
$$

To save space, we will not perform the final step in the analysis of the $O(\delta)$ system of equations, which involves integrating remaining terms in (5.12).

The most important part of the analysis above is the removal of all terms that are independent of $x^{*}$, since these terms lead to the homogenized equations. To see this, let us define

$$
\begin{gathered}
\underline{u}(x, t)=\left\langle u\left(x, x^{*}, t\right)\right\rangle=\underline{u}^{(0)}(x, t)+\delta \underline{u}^{(1)}(x, t)+O\left(\delta^{2}\right), \\
\underline{\sigma}(x, t)=\left\langle\sigma\left(x, x^{*}, t\right)\right\rangle=\underline{\sigma}^{(0)}(x, t)+\delta \underline{\sigma}^{(1)}(x, t)+O\left(\delta^{2}\right)
\end{gathered}
$$


and add up the homogenized equations (5.9) and (5.13) to get

$$
\begin{aligned}
0=\left\langle K^{-1}\right\rangle \underline{\sigma}_{t}-G\left(\underline{\sigma}^{(0)}\right) \underline{u}_{x}+\delta\left\langle\llbracket \rho \rrbracket K^{-1}\right\rangle \underline{u}_{t t}-\delta\left\langle\llbracket \rho \rrbracket K^{-1}\right\rangle \underline{u}_{t} \underline{\sigma}_{t} G^{\prime}\left(\underline{\sigma}^{(0)}\right) \\
G\left(\underline{\sigma}^{(0)}\right) \\
-\delta\left\langle K^{-1}\right\rangle \frac{\underline{\sigma}^{(1)} \underline{\sigma}_{t} G^{\prime}\left(\underline{\sigma}^{(0)}\right)}{G\left(\underline{\sigma}^{(0)}\right)}+O\left(\delta^{2}\right), \\
0=\langle\rho\rangle \underline{u}_{t}-\underline{\sigma}_{x}+\delta\left\langle\rho \llbracket K^{-1} \rrbracket\right\rangle \frac{G\left(\underline{\sigma}^{(0)}\right) \underline{\sigma}_{t t}^{(0)}-\left(\underline{\sigma}_{t}^{(0)}\right)^{2} G^{\prime}\left(\underline{\sigma}^{(0)}\right)}{G\left(\underline{\sigma}^{(0)}\right)^{2}}+O\left(\delta^{2}\right) .
\end{aligned}
$$

To make these equations look more like the original system of equations (3.3), we replace all $t$-derivatives (except for the first terms in (5.14)) with $x$-derivatives by substituting the equations into themselves. For example,

$$
\begin{aligned}
\frac{G\left(\underline{\sigma}^{(0)}\right) \underline{\sigma}_{t t}^{(0)}-\left(\underline{\sigma}_{t}^{(0)}\right)^{2} G^{\prime}\left(\underline{\sigma}^{(0)}\right)}{G\left(\underline{\sigma}^{(0)}\right)^{2}} & =\frac{\partial}{\partial t}\left[\frac{\underline{\sigma}_{t}^{(0)}}{G\left(\underline{\sigma}^{(0)}\right)}\right]=\frac{\partial}{\partial t}\left[\frac{\underline{u}_{x}^{(0)}}{\left\langle K^{-1}\right\rangle}\right]+O(\delta) \\
& =\frac{\underline{\sigma}_{x x}^{(0)}}{\langle\rho\rangle\left\langle K^{-1}\right\rangle}+O(\delta)=\frac{\underline{\sigma}_{x x}}{\langle\rho\rangle\left\langle K^{-1}\right\rangle}+O(\delta) .
\end{aligned}
$$

Similar substitutions eventually produce

$$
0=\left\langle K^{-1}\right\rangle \underline{\sigma}_{t}-G\left(\underline{\sigma}^{(0)}\right) \underline{u}_{x}-\delta \underline{\sigma}^{(1)} \underline{u}_{x} G^{\prime}\left(\underline{\sigma}^{(0)}\right)+\delta \frac{\left\langle\llbracket \rho \rrbracket K^{-1}\right\rangle}{\langle\rho\rangle\left\langle K^{-1}\right\rangle} G\left(\underline{\sigma}^{(0)}\right) \underline{u}_{x x}+O\left(\delta^{2}\right),
$$

$$
0=\langle\rho\rangle \underline{u}_{t}-\underline{\sigma}_{x}+\delta \frac{\left\langle\rho \llbracket K^{-1} \rrbracket\right\rangle}{\langle\rho\rangle\left\langle K^{-1}\right\rangle} \underline{\sigma}_{x x}+O\left(\delta^{2}\right) .
$$

Furthermore, we recognize the first few terms of the expansion of $G(\underline{\sigma})$ in (5.15a), and thus this can be simplified to

$$
\begin{aligned}
& 0=\left\langle K^{-1}\right\rangle \underline{\sigma}_{t}-G(\underline{\sigma}) \underline{u}_{x}+\delta \frac{\left\langle\llbracket \rho \rrbracket K^{-1}\right\rangle}{\langle\rho\rangle\left\langle K^{-1}\right\rangle} G(\underline{\sigma}) \underline{u}_{x x}+O\left(\delta^{2}\right), \\
& 0=\langle\rho\rangle \underline{u}_{t}-\underline{\sigma}_{x}+\delta \frac{\left\langle\rho \llbracket K^{-1} \rrbracket\right\rangle}{\langle\rho\rangle\left\langle K^{-1}\right\rangle} \underline{\sigma}_{x x}+O\left(\delta^{2}\right) .
\end{aligned}
$$

The equations above represent the homogenized versions of (3.3), with terms up to $O(\delta)$ included. However, $\left\langle\rho \llbracket K^{-1} \rrbracket\right\rangle=0$ for piecewise constant functions $\rho(x)$ and $K(x)$, so we have to compute more terms of this equation to see any interesting behavior. Hence the terms involving second derivatives vanish in this case, and dispersive effects at the next order will dominate. (For other choices of $\rho(x)$ and $K(x)$ that are rapidly varying but not piecewise constant, the second-derivative terms may not drop out. Numerical experiments show different behavior in this case, but we have not yet investigated this in detail.)

As the algebra involved increases exponentially with each order of $\delta$, we have employed Mathematica to perform the calculations. The homogenized equations in- 
cluding $O\left(\delta^{2}\right)$ terms are found to be

$$
\begin{array}{r}
\begin{aligned}
0=\left\langle K^{-1}\right\rangle \underline{\sigma}_{t}-G\left(\underline{\sigma} \underline{u}_{x}+\delta C_{11} G(\underline{\sigma}) \underline{u}_{x x}+\delta^{2} C_{12} G(\underline{\sigma}) \underline{u}_{x x x}\right. \\
+\delta^{2} C_{13}\left(G^{\prime}(\underline{\sigma}) \underline{\sigma}_{x} \underline{u}_{x x}+\frac{1}{2} G^{\prime \prime}(\underline{\sigma}) \underline{\sigma}_{x}^{2} \underline{u}_{x}\right)+O\left(\delta^{3}\right),
\end{aligned} \\
0=\langle\rho\rangle \underline{u}_{t}-\underline{\sigma}_{x}+\delta C_{21} \underline{\sigma}_{x x}+\delta^{2} C_{22} \underline{\sigma}_{x x x}+O\left(\delta^{3}\right),
\end{array}
$$

where

$$
\begin{aligned}
C_{11} & =\frac{\left\langle\llbracket \rho \rrbracket K^{-1}\right\rangle}{\langle\rho\rangle\left\langle K^{-1}\right\rangle}, \\
C_{21} & =\frac{\left\langle\rho \llbracket K^{-1} \rrbracket\right\rangle}{\langle\rho\rangle\left\langle K^{-1}\right\rangle}, \\
C_{12} & =\frac{\left\langle\llbracket K^{-1} \rrbracket \llbracket \rho \rrbracket\right\rangle}{\left\langle K^{-1}\right\rangle\langle\rho\rangle}-\frac{\left\langle K^{-1} \llbracket \rho \rrbracket^{2}\right\rangle}{\left\langle K^{-1}\right\rangle\langle\rho\rangle^{2}}, \\
C_{22} & =\frac{\left\langle\llbracket K^{-1} \rrbracket \llbracket \rho \rrbracket\right.}{\left\langle K^{-1}\right\rangle\langle\rho\rangle}-\frac{\left\langle\llbracket K^{-1} \rrbracket^{2} \rho\right\rangle}{\left\langle K^{-1}\right\rangle^{2}\langle\rho\rangle}, \\
C_{13} & =2 \frac{\left\langle\llbracket K^{-1} \rrbracket \rho\right\rangle^{2}}{\left\langle K^{-1}\right\rangle^{2}\langle\rho\rangle^{2}}+2 \frac{\left\langle\llbracket K^{-1} \rrbracket \llbracket \rho \rrbracket\right\rangle}{\left\langle K^{-1}\right\rangle\langle\rho\rangle}-2 \frac{\left\langle\llbracket K^{-1} \rrbracket^{2} \rho\right\rangle}{\left\langle K^{-1}\right\rangle^{2}\langle\rho\rangle}-\frac{\left\langle K^{-1} \llbracket \rho \rrbracket^{2}\right\rangle}{\left\langle K^{-1}\right\rangle\langle\rho\rangle^{2}} .
\end{aligned}
$$

In general it can be shown that $C_{11}=-C_{12}$; see [11]. For the special case of piecewise constant material parameters considered in this paper, we find that

$$
\begin{aligned}
& C_{11}=C_{21}=0, \\
& C_{12}=-\frac{1}{12} \alpha^{2}(1-\alpha)^{2} \frac{\left(\rho_{A}-\rho_{B}\right)\left(Z_{A}^{2}-Z_{B}^{2}\right)}{K_{A} K_{B}\left\langle K^{-1}\right\rangle\langle\rho\rangle^{2}}, \\
& C_{22}=-\frac{1}{12} \alpha^{2}(1-\alpha)^{2} \frac{\left(K_{A}-K_{B}\right)\left(Z_{A}^{2}-Z_{B}^{2}\right)}{K_{A}^{2} K_{B}^{2}\left\langle K^{-1}\right\rangle^{2}\langle\rho\rangle}, \\
& C_{13}=-\frac{1}{12} \alpha^{2}(1-\alpha)^{2} \frac{\langle\rho\rangle^{2}\left(K_{A}-K_{B}\right)^{2}+\left(Z_{A}^{2}-Z_{B}^{2}\right)}{K_{A}^{2} K_{B}^{2}\left\langle K^{-1}\right\rangle^{2}\langle\rho\rangle^{2}} .
\end{aligned}
$$

Here $Z_{A}=\sqrt{\rho_{A} K_{A}}$ and $Z_{B}=\sqrt{\rho_{B} K_{B}}$ are the linearized impedances. Note that if $Z_{A}=Z_{B}$, then $C_{12}=C_{22}=0$. Also note that for the linear case $G(\sigma)=1$, the factor multiplying $C_{13}$ vanishes in the homogenized equations (5.17).

Additional terms in the homogenized equations are too complicated to present in their general form. For the specific case used in section 3 to produce Figures 4-9, we have calculated the homogenized equations including $O\left(\delta^{4}\right)$ terms. We take $\alpha=1 / 2$ and use the exponential stress-strain relationship $G(\sigma)=\sigma+1$ and the piecewise constant material parameters $\rho_{A}=k_{A}=4, \rho_{B}=k_{B}=1$. The homogenized 
equations are then

$$
u_{t}=\frac{2 \sigma_{x}}{5}+\frac{3 \delta^{2} \sigma_{x x x}}{500}+\delta^{4}\left(\frac{3 \sigma_{x x x} \sigma_{x}^{2}}{15625(\sigma+1)^{2}}-\frac{72 u_{x x}^{2} \sigma_{x}}{15625(\sigma+1)}-\frac{12 \sigma_{x x x x} \sigma_{x}}{15625(\sigma+1)}\right.
$$

$$
\begin{gathered}
\left.-\frac{96 u_{x x} u_{x x x}}{15625}-\frac{12 \sigma_{x x} \sigma_{x x x}}{15625(\sigma+1)}-\frac{357 \sigma_{x x x x x}}{1000000}\right)+O\left(\delta^{6}\right), \\
\sigma_{t}=\frac{8(\sigma+1) u_{x}}{5}+\delta^{2}\left(\frac{3(\sigma+1) u_{x x x}}{125}+\frac{3 u_{x x} \sigma_{x}}{50}\right) \\
+\delta^{4}\left(\frac{48 u_{x} u_{x x}^{2}}{15625}-\frac{48 \sigma_{x} \sigma_{x x} u_{x x}}{15625(\sigma+1)}-\frac{4761 \sigma_{x x x} u_{x x}}{500000}-\frac{72 u_{x x x} \sigma_{x}^{2}}{15625(\sigma+1)}\right. \\
\left.(5.20 \mathrm{~b}) \quad-\frac{357(\sigma+1) u_{x x x x x}}{250000}-\frac{3543 u_{x x x x} \sigma_{x}}{500000}-\frac{3891 u_{x x x} \sigma_{x x}}{500000}\right)+O\left(\delta^{6}\right) .
\end{gathered}
$$

(The underlines have been dropped for clarity.) Note again that for this case the $u_{x x}$ and $\sigma_{x x}$ terms drop out, and we can see the appearance of dispersive terms like $u_{x x x}$ and $\sigma_{x x x}$ in $\delta^{2}$ terms. Also notice that (5.20) has the property that it is invariant under the reflection $u(x, t) \rightarrow-u(-x, t)$ and $\sigma(x, t) \rightarrow \sigma(-x, t)$, a property inherited from the original equations (3.3).

Furthermore, we see that each term proportional to $\delta^{n}$ always has a total of $n+1$ spatial derivatives, which corroborates the observation made earlier that if $\tilde{u}(x, t)$ and $\tilde{\sigma}(x, t)$ are solutions to (5.20) for $\delta=1$, then $u(x, t)=\tilde{u}(x / \delta, t / \delta)$ and $\sigma(x, t)=\tilde{\sigma}(x / \delta, t / \delta)$ are solutions for arbitrary $\delta$. This property implies that the value of $\delta$ does not have to be small and that these homogenized equations are valid for any $\delta$. This suggests that the "higher-order" terms can be dropped, not because $\delta$ is small, but because they involve small coefficients coming from higher-order averages of the rapidly varying coefficients, as is already apparent in (5.20).

In Figure 12 we compare the solution to the homogenized equations (5.20) with $\delta=1$, dropping the $O\left(\delta^{6}\right)$ terms, with the numerical solution to the original equations (3.3). The homogenized equations were solved using a pseudospectral numerical method that computes these smooth solutions with high accuracy (using techniques discussed in [2] and [9] for similar equations). In this test we take initial data consisting of a nonzero strain in the middle of the computational domain, with zero velocity, which results in two outgoing pulses that each break up into a train of stegotons. At later times we show only the leftgoing wave train. We use this initial data with periodic boundary conditions for ease in using the pseudospectral method, and also because it is not clear how to impose appropriate boundary conditions of the type used earlier for the higher-order homogenized equations. The agreement is good, especially when one considers that the waves have traveled over 400 units by $t=500$. If only the terms up to $O\left(\delta^{3}\right)$ are retained, solitary waves are still observed, but the agreement is not as good.

Solving the homogenized equations with boundary data corresponding directly to the layered medium tests done earlier would require the derivation of appropriate boundary conditions for these higher-order equations. An example of how this can be done in a weakly nonlinear case is shown in [10] and [11], but this has not yet been carried through for the homogenized equations presented here.

6. Conclusions. We have studied elastic waves in nonlinear layered media by computing numerical solutions to a first-order hyperbolic system of conservation laws 

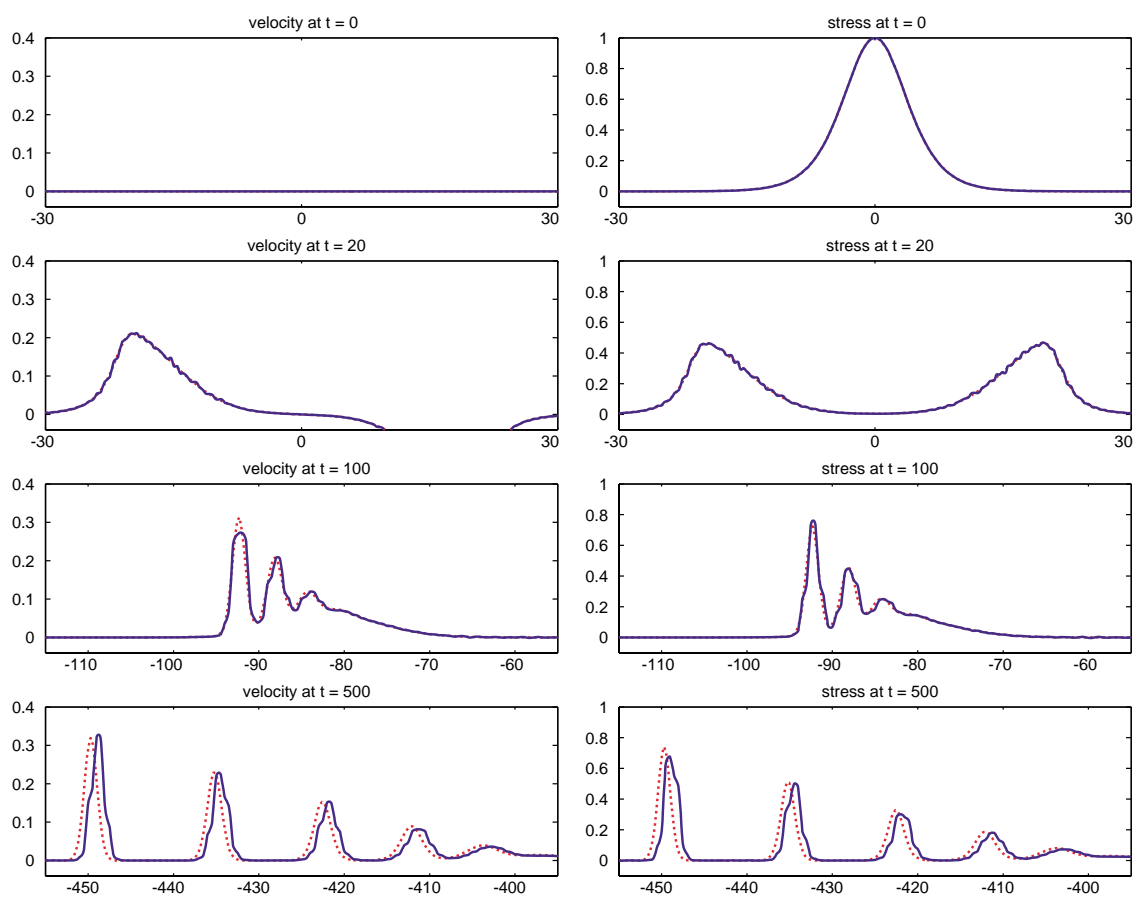

FIG. 12. Comparison of a pseudospectral solution (dashed curve) to the homogenized equations (5.20) with the finite-volume solution (solid curve) to the layered medium equations (3.3).

with a spatially varying flux function, using the method described in [1] and [4]. The layering leads to dispersive behavior if the layers are not impedance-matched, which in turn gives rise to the appearance of solitary waves that appear to interact in the manner of classical solitons. There is an approximate one-parameter family of such waves, whose velocity varies linearly with amplitude. By studying the solution as a function of time at a fixed point $x_{0}$, we obtain some indication of the scaling properties of these waves as the amplitude is varied.

We also showed that, for a special limiting choice of parameters, the layered medium can be modeled directly by the Toda lattice. Since the Toda lattice is known to have exact soliton solutions, it is not surprising that similar behavior is observed in the layered medium in this case. It is more surprising that solitary waves are observed in cases far from this limit.

A set of nonlinear homogenized equations has been derived that contains dispersive terms. Numerical solution of these equations yields results that agree well with the direct solution of the original hyperbolic system. We hope that further study of these equations may provide more insight into the nature of these solitary waves.

We have studied in detail only one particular choice of material parameters in the piecewise constant case with an exponential stress-strain relation. Preliminary experiments with different choices show a rich variety of other interesting behavior that should be explored further.

Acknowledgment. The authors would like to thank Arnold D. Kim for advice on pseudospectral numerical methods. 


\section{REFERENCES}

[1] D. S. Bale, R. J. LeVeque, S. Mitran, And J. A. Rossmanith, A wave propagation method for conservation laws and balance laws with spatially varying flux functions, SIAM J. Sci. Comput., 24 (2002), pp. 955-978.

[2] T. B. Benjamin, J. L. Bona, And J. J. Mahony, Model equations for long waves in nonlinear dispersive systems, Philos. Trans. Roy. Soc. London Ser. A, 272 (1972), pp. 47-78.

[3] J. KeVORKIAN AND D. L. Bosley, Multiple-scale homogenization for weakly nonlinear conservation laws with rapid spatial fluctuations, Stud. Appl. Math., 101 (1998), pp. 127-183.

[4] R. J. LEVEQUE, Finite volume methods for nonlinear elasticity in heterogeneous media, Internat. J. Numer. Methods Fluids, 40 (2002), pp. 93-104.

[5] A. M. Samsonov, Strain Solitons in Solids and How to Construct Them, Chapman \& Hall/ CRC, Boca Raton, FL, 2001.

6] F. Santosa And W. W. Symes, A dispersive effective medium for wave propagation in periodic composites, SIAM J. Appl. Math., 51 (1991), pp. 984-1005.

[7] M. TodA, Nonlinear Waves and Solitons, Kluwer Academic Publishers, Boston, 1989.

[8] M. TodA, Theory of Nonlinear Lattices, Springer-Verlag, New York, Berlin, 1989.

[9] L. N. Trefethen, Spectral Methods in MATLAB, Software Environ. Tools. 10, SIAM, Philadelphia, 2000.

[10] D. H. Yong, Solving Boundary-Value Problems for Systems of Hyperbolic Conservation Laws with Rapidly Varying Coefficients, Ph.D. dissertation, University of Washington, Seattle, 2000.

[11] D. H. YONG AND J. KEVORKIAN, Solving boundary-value problems for systems of hyperbolic conservation laws with rapidly varying coefficients, Stud. Appl. Math., 108 (2002), pp. 259303. 